\title{
A Inserção da Álgebra Linear no Currículo da Primeira Universidade Brasileira
}

\author{
How Linear Algebra Entered the Curriculum of the First Brazilian \\ University
}

\author{
Gabriel Loureiro de Lima* \\ ORCID iD 0000-0002-5723-0582 \\ Eloiza Gomes** \\ ORCID iD 0000-0002-1217-9904
}

\begin{abstract}
Resumo
Principalmente em consequência do esforço despendido por cientistas durante os séculos XVII a XIX visando construir um sistema de cálculo intrínseco aos elementos geométricos que viabilizasse a aplicação do método analítico à solução de problemas da Geometria, a Álgebra Linear pôde consolidar-se no início do século XX como um novo ramo da Matemática. Partindo dessa premissa, evidenciamos neste artigo o modo como se deu sua introdução como conteúdo de ensino e, posteriormente, como disciplina acadêmica universitária na graduação em Matemática e nos cursos de Engenharia da Universidade de São Paulo (USP). Destacamos a influência exercida nesse processo por matemáticos franceses, especialmente alguns daqueles que constituíam o grupo Bourbaki e que estiveram presentes na USP, de 1945 a 1966. No curso de Matemática da Faculdade de Filosofia, Ciências e Letras da USP, desde o início da década de 1950 até 1964, a Álgebra Linear esteve presente como conteúdo de ensino em Complementos de Geometria. Somente em 1965 é que, pela primeira vez, comparece no currículo desse curso uma disciplina intitulada Álgebra Linear. Já nos cursos de Engenharia da Escola Politécnica de São Paulo, a Álgebra Linear surge em 1961 efetivamente como disciplina, momento em que aquela que até então se chamava Elementos de Geometria Analítica e Projetiva, e que desde 1958 contemplava a Álgebra Linear como conteúdo de ensino, foi reintitulada Elementos de Álgebra Linear e Geometria Analitica.
\end{abstract}

Palavras-chave: Álgebra Linear. História da Educação Matemática. Grupo Bourbaki. Universidade de São Paulo. Escola Politécnica de São Paulo.

\begin{abstract}
A major factor resulting in the consolidation of linear algebra as a new branch of mathematics in the early 20th century was the efforts made by 18th and 19th-century scientists to develop a system of calculation that, intrinsic to geometric elements, allowed geometry problems to be solved by using the analytical method. Drawing on this premise, we describe in this article the process by which linear algebra was initially introduced as a teaching content, before gaining the academic course status, into the undergraduate programs in mathematics and engineering at the University of São Paulo (USP). Particularly relevant to this process was the role played by

\footnotetext{
* Doutor em Educação Matemátca pela Pontifícia Universidade Católica de São Paulo (PUC/SP). Professor do Programa de Estudos Pós-Graduados em Educação Matemática da Pontifícia Universidade Católica de São Paulo (PUC/SP), São Paulo, São Paulo, Brasil. Endereço para correspondência: Rua Marquês de Paranaguá, 111, Consolação, São Paulo, SP, Brasil, CEP: 013013-050. E-mail: gllima@pucsp.br.

** Doutora em Educação Matemátca pela Pontifícia Universidade Católica de São Paulo (PUC-SP). Professor Associado do Centro Universitário do Instituto Mauá de Tecnologia (CEUN-IMT), São Caetano do Sul, São Paulo, Brasil. Endereço para correspondência: Praça Mauá, 1, Bairro Mauá, São Caetano do Sul, São Paulo, Brasil, CEP: 09580-900. E-mail: eloiza@ maua.br.
} 
French mathematicians, especially members of the Bourbaki Group, who taught at USP during the 1945-1966 period. In the mathematics program of USP's School of Philosophy, Sciences, and Languages, linear algebra was a teaching content on the Geometry Complements course from the early 1950s up until 1964. Only in 1965 was a course entitled Linear Algebra introduced into the program's curriculum. In the São Paulo Polytechnic School's Engineering Programs, however, linear algebra had been taught since 1961 as a full-fledged course called Elements of Linear Algebra and Analytical Geometry, renaming the course previously called Elements of Analytical and Projective Geometry, in which linear algebra had constituted a teaching content since 1958.

Keywords: Linear algebra. History of Mathematics Education. Bourbaki Group. University of São Paulo. Polytechnic School of São Paulo.

\section{Introdução}

Ao analisarmos, tomando como principal referência o estudo de Dorier (1990), os processos de desenvolvimento histórico da Geometria Analítica e da Álgebra Linear, percebemos que essas duas subáreas da Matemática se desenvolveram conjuntamente a partir do momento em que se buscou aplicar o método analítico à resolução de problemas geométricos.

Questionamo-nos então como as disciplinas de Geometria Analítica e de Álgebra Linear, estando tais campos consolidados, se relacionaram ao longo do tempo no Ensino Superior. Passamos a buscar informações a esse respeito, analisando programas de ensino dos primeiros anos de funcionamento do curso de Matemática da Universidade de São Paulo (USP) e alguns da Escola Politécnica de São Paulo (Poli), criada em 1893 e incorporada à USP em 1934, anteriores à fundação da referida universidade.

Por meio de tal análise, verificamos que somente a Geometria Analítica constava em tais programas, sem que os conteúdos da Álgebra Linear estivessem presentes nem mesmo em outras disciplinas. Questionamo-nos, então, quando se teria começado a ensinar Álgebra Linear na USP, primeira universidade do país, que, por muitas décadas, foi modelo para outras instituições e, consequentemente, para cursos posteriormente criados. Longas pesquisas não nos revelaram tal informação. Apenas indícios pulverizados constavam em diferentes estudos.

Frente a essa constatação, optamos por nos dedicar a uma investigação de caráter bibliográfico que nos permitisse compreender esse processo de constituição, na primeira universidade brasileira, da Álgebra Linear como disciplina acadêmica universitária ${ }^{1}$. Para a coleta dos dados, recorremos principalmente a dissertações, teses e artigos que versavam sobre os primeiros anos de funcionamento da Poli e também da USP, bem como Guias da Faculdade

\footnotetext{
${ }^{1}$ Assim como Resende (2007, p. 77), entendemos por disciplina acadêmica universitária um "conjunto de conteúdos e práticas, frutos de uma transposição didática, incluindo finalidades, elementos pedagógicos e outros do meio profissional de referência e da sociedade em geral, organizado de modo a manter uma unidade científica e didática".
} 
de Filosofia, Ciências e Letras da USP e, finalmente, livros de Álgebra e de Álgebra Linear, tanto brasileiros quanto estrangeiros, de diferentes épocas.

Partimos da premissa de que, principalmente como consequência do esforço despendido por cientistas nos séculos XVII a XIX, visando a construção de um sistema de cálculo intrínseco aos elementos geométricos que viabilizasse a aplicação do método analítico à solução de problemas da Geometria, a Álgebra Linear pôde consolidar-se, no início do século XX, como um novo ramo da Matemática. Tal foco permitiu-nos evidenciar nesse artigo como se deu o ingresso da Álgebra Linear como conteúdo de ensino e, posteriormente, como disciplina acadêmica universitária na graduação em Matemática e nos cursos de Engenharia da USP.

Como detalharemos ao longo deste artigo, tal inserção se deu por influência de matemáticos franceses, especialmente daqueles que constituíam o grupo Bourbaki. Integrantes desse grupo estiveram presentes na USP, de 1945 a 1966, quando influenciaram a inserção de novas disciplinas - dentre elas a Álgebra Linear - tanto no currículo da Faculdade de Filosofia, Ciências e Letras (FFCL, onde funcionava o curso de Matemática), quanto no da Poli.

Visando compreender como se deu a influência francesa no curso de Matemática da USP e na Poli, apresentaremos inicialmente algumas informações a respeito da introdução da Álgebra Linear como disciplina no currículo francês.

\section{A inserção da Álgebra Linear no ensino francês}

Segundo Dorier (1990), é difícil precisar quando o ensino de Álgebra Linear foi introduzido na França em sua forma moderna. Certamente isso ocorreu por iniciativa de professores universitários que começaram a trabalhá-la com os alunos dos anos finais dos cursos de Graduação, bem como na Pós-Graduação.

Os anos 1930 marcaram o início de uma mudança fundamental no ensino de Matemática nas universidades francesas, de acordo com Dorier (2002), que relata que jovens matemáticos da École Normale Supérieure descobriram e passaram a valorizar o livro alemão Moderne algebra, de autoria do holandês Bartel Leendert van der Waerden (1903-1996), que o estruturou, como explicita Oliveira (2007), a partir de lições da alemã Emmy Noether (18821935) e do austríaco Emil Artins (1898-1962), tendo o primeiro volume sido lançado em 1930 e o segundo em 1931.

Até então, como aponta Dorier (2002), essa obra fora totalmente ignorada pelos matemáticos mais antigos da École Normale Supérieure, até que esses jovens perceberam, por meio desse livro, uma nova maneira de fazer Matemática, "não somente pela modernidade dos 
temas tratados, mas também por se caracterizar por uma apresentação concisa, rigorosa, que organiza os diversos objetos da Álgebra segundo um desenvolvimento estrutural” (p. 59).

Ao concluírem seus estudos e, pouco a pouco, tornarem-se também professores universitários, alguns desses jovens, como ressalta Dorier (2002), sentiram a necessidade de renovar o ensino. Em 1940, por exemplo, Henri Cartan (1904-2008) tornou-se professor na Universidade de Clermont-Ferrand, em Estrasburgo, onde ministrou um curso completamente novo, que se iniciava pela apresentação axiomática de conceitos algébricos e topológicos e voltava atenção às estruturas, incluindo nesse contexto a teoria dos espaços vetoriais.

No ano seguinte, Cartan foi nomeado professor da École Normale Supérieure e René de Possel (1905-1974) o substituiu em Clermont-Ferrand, ali ministrando um curso similar. Também em 1940 e 1941, na mesma universidade, Charles Ehresmann (1905-1979), em seu curso de cinemática, apresentava um tratamento axiomático de vetor em dimensão finita e trabalhava com espaços afins e transformações lineares.

Em 1934, um grupo de matemáticos franceses, incluindo muitos dos que buscavam renovar o ensino de Matemática em universidades francesas, como os já citados Cartan e Possel, além de outros como Jean Dieudonné (1906-1992) e André Weil (1906-1998), se reuniu sob o pseudônimo coletivo Nicolas Bourbaki.

Oliveira (2007, p. 59) esclarece que nos manuais franceses, como, por exemplo, o Cours d'analyse, de Édouard Jean-Baptiste Goursat (1858-1936), “os temas clássicos da Análise são tratados de maneira detalhada, o que resulta num texto prolixo, no qual as ideias gerais mal aparecem. O rigor deixa a desejar, os teoremas se repetem a cada vez com hipóteses suplementares". Foi nesse sentido que, inspirado pelo estilo adotado por van der Waerden em seu Moderne algebra,

O grupo Bourbaki se propôs a redigir um tratado moderno de Análise Matemática que cuidasse da apresentação, reforçasse o rigor, incluísse os desenvolvimentos mais recentes. [...] Seus talentos aliados à diversidade de suas experiências e especialidades, a influência da Álgebra alemã, sua vontade de clarear e ampliar os conceitos contribuíram para que o grupo ultrapassasse a simples missão de escrever um novo tratado de Análise. Eles não se ocuparam unicamente de renovar a Análise, mas a Matemática, em geral (OLIVEIRA, 2007, p. 59).

Assim como na obra de van der Waerden, o grupo Bourbaki buscou uma abordagem estrutural da Matemática. Dieudonné (1990), como menciona Pires (2006), considera que o aspecto central de tal abordagem é a ideia de que aquilo que efetivamente desempenha papel fundamental em uma teoria são as relações entre os objetos matemáticos e não a natureza de tais objetos. O matemático, "uma vez que haja discernido, nos elementos que estuda, relações que satisfazem os axiomas de uma estrutura conhecida, dispõe de imediato de todo o arsenal de 
teoremas gerais relativos às estruturas desse tipo" (PIRES, 2006, p. 144).

Em 1947, o grupo Bourbaki publicou o capítulo 2 (intitulado Álgebra linear), do livro II (Álgebra), da primeira parte (As estruturas fundamentais da análise), de seus Elementos de matemática. Essa obra rapidamente se tornou referência em muitos domínios da Matemática e exerceu grande influência no ensino dessa ciência, principalmente na França, mas também no restante do mundo, ganhando notoriedade em diferentes países e influenciando professores e autores de livros didáticos na maneira de apresentar os conteúdos e no vocabulário empregado.

\begin{abstract}
Uma característica importante da obra de Bourbaki é que o modo de exposição é axiomático e segue do mais geral para o particular. Não há muitos exemplos e os poucos vêm somente depois da apresentação axiomática. A linguagem é econômica e concisa, contendo notas históricas ao final de cada capítulo. Os exercícios têm duplo objetivo: um que é o habitual de levar o leitor a verificar se entendeu o texto, o outro que é fazer conhecer resultados que não tiveram lugar no texto. São deixados como exercícios vários resultados interessantes (e importantes) que constituem aplicações, mas que não se inserem no desenvolvimento estritamente lógico do texto (OLIVEIRA, 2007, p. 60).
\end{abstract}

Conforme destaca na introdução de seus Elementos de matemática, o grupo Bourbaki justificava a importância do estudo da Álgebra Linear pelo fato de esta guardar profunda relação tanto com a Geometria clássica, quanto com a Análise Matemática moderna.

Dorier (1990) salienta que foram as orientações bourbakistas que guiaram as primeiras tentativas francesas de ensino de Álgebra moderna, em particular de Álgebra Linear. Ressalva, no entanto, que o grupo Bourbaki não foi o único a promover na França a teoria axiomática dos espaços vetoriais.

Em 1935, por exemplo, Gaston Maurice Julia (1893-1978) proferiu para físicos e matemáticos uma série de conferências na Universidade Sorbonne, tratando das bases matemáticas para a Mecânica Quântica. Nelas apresentou em detalhes a teoria de espaços vetoriais euclidianos de dimensão finita, inicialmente de maneira clássica, recorrendo a coordenadas e determinantes e, posteriormente, por meio de uma abordagem axiomática, ressaltando as vantagens desta última.

Como salienta Dorier (1990), foi somente na década de 1950 que tal conteúdo começou a ser abordado também no primeiro ciclo francês (que abrange os dois primeiros anos da Graduação). Dorier (2002) também destaca que um passo decisivo para a renovação do ensino universitário francês foi dado em 1954, quando Gustave Choquet (1915-2006), jovem matemático respeitado pelos membros do grupo Bourbaki, tornou-se o responsável pelo ensino de Matemática para o primeiro ciclo da Universidade de Paris, onde impôs uma mudança radical, introduzindo uma abordagem estrutural axiomática para essa ciência. Rapidamente, essa "revolução parisiense" se espalhou por toda a França e, nesse contexto, a teoria dos espaços 
vetoriais assumiu papel central, contribuindo para a reformulação do ensino da Geometria.

Em 1963, a Associação Nacional de Professores de Matemática (APMEP) publicou, segundo Dorier (2002), um livro dedicado à teoria dos espaços vetoriais de dimensões finitas. A obra, inspirada nas ideias do grupo Bourbaki, tornou-se referência para o ensino da Álgebra Linear na França.

Dorier (1990, p. 83) destaca que, em 1967, um texto oficial estabeleceu o programa mínimo de Matemática a ser trabalhado nos primeiros anos de Graduação nas Faculdades de Ciência, da França. No âmbito da Álgebra Linear, previam-se estudos relacionados aos espaços sobre os corpos dos números reais e dos complexos, contemplando os seguintes tópicos:

- espaço e subespaço vetorial, espaço gerado, dependência e independência linear, espaço de dimensão finita, base;

- aplicação linear, núcleo, imagem, posto, espaços de aplicações lineares, composição de aplicações lineares;

- cálculo matricial;

- formas multilineares alternadas de grau máximo, determinantes;

- equações lineares.

O autor salienta que nos livros franceses da época a apresentação dos conceitos de Álgebra Linear, mais uma vez, seguia o esquema da obra de Bourbaki.

Embora não esteja especificamente relacionada à inserção da Álgebra Linear no currículo francês, a obra Survey of modern algebra, dos matemáticos estadunidenses Garrett Birkhoff (1911-1996) e Saunders MacLane (1909-2005), publicada em 1941, merece destaque, segundo Dorier (1990), por ter levado o estudo da Álgebra moderna também para o continente americano.

De acordo com Gouvêa (2010), responsável por uma resenha de Survey of modern algebra, a obra foi elaborada tendo-se em mente a necessidade de produzir um livro de Álgebra, em abordagem moderna, que fosse acessível aos alunos de Graduação. Gouvêa destaca também que Birkhoff e MacLane, ao contrário de outros autores, não redigiram um manual adotando a mesma relação de conteúdos e a mesma sequência proposta por van der Waerden em seu Moderne algebra, referência para o ensino universitário desse ramo da Matemática até então. Buscaram, efetivamente, uma organização que tornasse o conteúdo mais acessível.

O espaço ocupado pela Álgebra Linear nesse manual é considerável e a apresentação é bem semelhante à utilizada em muitos livros atuais. No prefácio, os autores destacam que a obra foi redigida de maneira a "não perder de vista o fato de que, para muitos estudantes, o valor da Álgebra reside em suas aplicações em outros campos: Análise, Geometria, Física e Filosofia. Isso nos influenciou na ênfase que damos ao trabalho nos domínios dos números reais 
e dos complexos" (BIRKHOFF; MACLANE, 1941, p. v; tradução nossa). Em relação, especificamente, aos conteúdos de Álgebra Linear, os autores especificam que:

\begin{abstract}
O tema matrizes e suas aplicações é de grande importância; nós nos esforçamos para dar-lhe o devido valor sem permitir, no entanto, que esse tema ofuscasse os outros tópicos. É desejável ter domínio não somente das operações formais com matrizes, mas também da interpretação (frequentemente negligenciada) das matrizes em termos das transformações lineares. Temos mantido esta interpretação geométrica continuamente em vista, enfatizando as propriedades pertinentes dos espaços vetoriais (BIRKHOFF; MACLANE, 1941, p. vi; tradução nossa).
\end{abstract}

Em 1967, consciente dos progressos ocorridos na área desde 1941, Birkhoff e MacLane publicam uma nova obra também voltada a cursos de Graduação, intitulada Algebra, e, em 1970, tal publicação ganhou versão francesa, com prefácio do bourbakista Dieudonné.

Dieudonné, a propósito, é personagem de relevo nesse processo de inserção da Álgebra Linear no currículo francês. Publicou em 1964 Algèbre linéaire et géométrie élémentaire, livro em que defendeu o estudo desse conteúdo, ainda que de maneira introdutória, também no Ensino Secundário destinado a estudantes que, posteriormente, ingressariam em cursos universitários da área de Ciências Exatas.

De acordo com Duarte (2007, p. 82-84), com base no que afirma Dieudonné (1973) na introdução de sua obra, o Ensino Secundário tradicional

[...] oferecia uma série de disciplinas, tais como geometria analítica, geometria
projetiva, teoria dos números complexos, trigonometria, etc., que eram apresentadas
separadamente e, com frequência, ignorando-se totalmente umas às outras e que
poderiam ser ensinada em uma só disciplina, a Álgebra Linear [...]. Uma das
vantagens da Álgebra Linear é que ela permitia apresentar a Geometria Elementar de
maneira rigorosa e sem esforços, ao passo que o estudo axiomático da Geometria
Euclidiana mostrava-se tão complexo e sutil, que eram apresentados aos alunos nada
mais do que "pseudo-raciocínios". O autor adotou como critério de elaboração de sua
obra desenvolver um programa destinado aos dois ou três últimos anos do secundário,
expondo somente tópicos que interessavam aos programas universitários, sem,
entretanto, procurar introduzir prematuramente teorias a serem ensinadas no ensino
superior. Procurou limitar-se às ideias que tinham importância não apenas à
Matemática como também à Física teórica.

A ideia de introduzir a Álgebra Linear no Ensino Secundário francês não agradou, de acordo com Dorier (1990), à comunidade matemática inicialmente, mas tornou-se realidade no final dos anos 1960, quando os preceitos da Matemática Moderna ganharam força nesse nível educacional e nele ocasionaram significativa reforma. Como evidenciam programas de ensino apresentados por esse autor, tal conteúdo começou a aparecer de maneira explícita nos currículos do Secundário por volta 1969, até que em meados da década de 1980 foi pouco a pouco perdendo espaço. Conforme informações colhidas no site do Ministère de l'Éducation Nationale de l'Enseignement Supérieur et de la Recherche ${ }^{2}$ da França, atualmente a Álgebra

\footnotetext{
${ }^{2}$ Disponível em: <http://eduscol.education.fr/pid26017/programmes-lycee.html>. Acesso em: 5 abr. 2017.
} 
Linear não está presente no Ensino Secundário.

Vejamos então como se deu, após a fundação da USP, a vinda ao Brasil de alguns membros do grupo Bourbaki e como as ideias difundidas por esses matemáticos, nos cursos e seminários que ministraram na FFCL dessa universidade, culminaram na introdução do ensino de Álgebra Linear também nos currículos universitários brasileiros.

\section{A presença de integrantes do grupo Bourbaki na FFCL da USP e a inserção da Álgebra Linear no Ensino Superior brasileiro}

O curso de Matemática implantado na FFCL da USP, em 1934, foi o primeiro destinado à formação de matemáticos e de professores de Matemática no país. Até então, esse papel era em parte exercido pelas escolas do Exército e da Marinha e pelas de Engenharia. Como ressalta Ziccardi (2009, p. 48), "antes da criação das Faculdades de Filosofia, o que se fez, no terreno da pesquisa matemática, foi resultado do esforço autodidata dos engenheiros com inclinação matemática”. Além disso, Lima (2012) relata que, até a fundação da USP, os professores de Matemática que atuavam na Educação Básica ou nas instituições de Ensino Superior já em funcionamento, ministrando Álgebra Superior, Cálculo, Geometria Analítica etc., eram engenheiros ou alunos dos últimos anos das escolas de Engenharia e oficiais do Exército ou da Marinha.

Para assumir as cadeiras de Ciências Exatas da FFCL, foram convidados, principalmente, professores de universidades italianas. Para a Matemática, as contratações de maior destaque foram as de Luigi Fantappiè (1901-1956) e Giacomo Albanese (1890-1947), que se tornaram responsáveis, respectivamente, pelas cadeiras de Análise Matemática e de Geometria.

Passaremos então a apresentar considerações a respeito da presença e atuação na FFCL da USP, em determinado período, de alguns membros do grupo Bourbaki.

\subsection{A presença de integrantes do grupo Bourbaki na FFCL da USP}

Pires (2006), em tese de doutorado intitulada A presença de Nicolas Bourbaki na Universidade de São Paulo, relata que, de 1945 a 1966, o Departamento de Matemática da FFCL da USP recebeu os seguintes membros do grupo Bourbaki: André Weil, Jean Dieudonné, Jean Delsarte (1903-1968), Samuel Eilenberg (1913-1998), Laurent Schwartz (1915-2002), Charles Ehresmann (1905-1979), Alexander Grothendieck (1928-2004) e Jean-Louis Koszul 
(1921-2018). As contratações incluíram também Oscar Zariski (1899-1986), que, embora não fosse bourbakista, mantinha estreita relação profissional com tal grupo.

A vinda de tais matemáticos foi incentivada pelo Departamento de Matemática, pois, com a eclosão da Segunda Guerra Mundial, em 1939, e o consequente retorno à Europa dos professores que haviam sido contratados na época da fundação da USP, havia a preocupação do departamento em dar continuidade ao estabelecimento de uma efetiva comunidade científica no país e, para isso, a contribuição de professores e pesquisadores internacionais de renome era fundamental. De fato, como apontou Omar Catunda (1906-1986) em reunião da Congregação da FFCL realizada em 5 de maio de 1942 e citada por Pires (2006, p. 228), naquele momento a elite intelectual brasileira estava ainda apenas em formação.

Durante sua estada na USP, esses membros do grupo Bourbaki e também Zariski ministraram cursos e conferências e colaboraram na orientação de trabalhos de doutoramento. Destacamos no Quadro 1, com base em dados trazidos por Pires (2006), as principais atividades por eles desenvolvidas de 1945 a 1958, evidenciando-se que a introdução da Álgebra Linear, inicialmente como conteúdo de ensino e posteriormente como disciplina no curso de Matemática da USP e nas graduações em Engenharia da Escola Politécnica de São Paulo, foi consequência direta das atividades desenvolvidas por esses integrantes do grupo Bourbaki, que aqui estiveram nas décadas de 1940 a 1960, e da influência exercida por tais atividades sobre os matemáticos brasileiros que lecionavam na FFCL e na Poli.

\begin{tabular}{|c|c|c|c|}
\hline Ano & Docente & Atividade & Título da atividade \\
\hline 1945 & André Weil & $\begin{array}{l}\text { Curso (na disciplina Análise } \\
\text { Superior) }\end{array}$ & $\begin{array}{c}\text { Formas Diferenciais, teoremas de } \\
\text { Rham e Espaços de Hilbert }\end{array}$ \\
\hline 1945 & Oscar Zariski & Curso & $\begin{array}{c}\text { Álgebra Moderna e Introdução à } \\
\text { Geometria Algébrica }\end{array}$ \\
\hline 1946 & André Weil & $\begin{array}{c}\text { Curso } \\
\text { (na disciplina Análise } \\
\text { Superior) } \\
\end{array}$ & Topologia Geral e Grupos Topológicos \\
\hline 1946 & Jean Dieudonné & Curso & Álgebra Moderna e Grupos de Galois \\
\hline 1947 & André Weil & $\begin{array}{c}\text { Curso } \\
\text { (na disciplina Análise } \\
\text { Superior) }\end{array}$ & Integrais Abelianas \\
\hline 1947 & Jean Delsarte & $\begin{array}{c}\text { Orientação da tese de } \\
\text { doutoramento de Elza Furtado } \\
\text { Gomide, com tema sugerido } \\
\text { por André Weil }\end{array}$ & Sobre o Teorema de Artin-Weil \\
\hline 1947 & Jean Dieudonné & Curso & Topologia Plana \\
\hline 1948 & Jean Delsarte & Curso & Teoria das Distribuições \\
\hline 1949 & Jean Delsarte & Curso & $\begin{array}{c}\text { Espaços Vetoriais Topológicos e Teoria } \\
\text { da Integração }\end{array}$ \\
\hline 1951 & Jean Delsarte & Curso & Hipergrupos e Álgebras de Lie \\
\hline 1951 & Oscar Zariski & $\begin{array}{c}\text { Orientação da tese de } \\
\text { doutoramento de Luiz } \\
\text { Henrique Jacy Monteiro }\end{array}$ & $\begin{array}{l}\text { Sobre as Potências Simbólicas de um } \\
\text { Ideal Primo de um Anel de Polinômios }\end{array}$ \\
\hline 1952 & $\begin{array}{c}\text { Samuel } \\
\text { Eilenberg }\end{array}$ & Curso & Topologia Algébrica \\
\hline
\end{tabular}




\begin{tabular}{|c|c|c|c|}
\hline 1952 & $\begin{array}{c}\text { Laurent } \\
\text { Schwartz }\end{array}$ & Conferência & Sobre a Teoria das Distribuições \\
\hline 1952 & $\begin{array}{c}\text { Laurent } \\
\text { Schwartz }\end{array}$ & Conferência & Funções Medioperiódicas \\
\hline 1952 & Jean Dieudonné & Conferência & Grupos Clássicos \\
\hline 1952 & Jean Dieudonné & Conferência & A Matemática na Antiga Grécia \\
\hline 1952 & $\begin{array}{c}\text { Charles } \\
\text { Ehresmann }\end{array}$ & Conferência & Espaços Fibrados \\
\hline $\begin{array}{l}\text { Entre } 1953 \text { e } \\
1955\end{array}$ & $\begin{array}{c}\text { Alexander } \\
\text { Grothendieck }\end{array}$ & Curso & Espaços Vetoriais Topológicos \\
\hline $\begin{array}{l}\text { Entre } 1954 \text { e } \\
1955\end{array}$ & $\begin{array}{l}\text { Jean-Louis } \\
\text { Koszul }\end{array}$ & Conferência & Faisceaux et Cohomologie \\
\hline 1958 & $\begin{array}{c}\text { Jean-Louis } \\
\text { Koszul }\end{array}$ & Seminário & Espaços Métricos \\
\hline $\begin{array}{l}\text { Entre } 1960 \text { e } \\
1968\end{array}$ & André Weil & \multicolumn{2}{|c|}{$\begin{array}{c}\text { Atuação como pesquisador no Instituto de Pesquisas Matemáticas, } \\
\text { fundado na USP, em } 1960\end{array}$} \\
\hline $\begin{array}{c}\text { Entre } 1960 \text { e } \\
1968 \\
\end{array}$ & $\begin{array}{c}\text { Charles } \\
\text { Ehresmann }\end{array}$ & \multicolumn{2}{|c|}{$\begin{array}{c}\text { Atuação como pesquisador no Instituto de Pesquisas Matemáticas, } \\
\text { fundado na USP, em } 1960\end{array}$} \\
\hline
\end{tabular}

Quadro 1 - Atividades desenvolvidas na USP por membros do grupo Bourbaki

Fonte: Dados da pesquisa, com base em Pires (2006).

\subsection{A inserção da Álgebra Linear, inicialmente como conteúdo de ensino e posteriormente como disciplina acadêmica universitária, no curso de Matemática da FFCL da USP e nas engenharias da Poli}

Nos primeiros anos do curso de Matemática da USP, os professores Fantappiè e Albanese organizaram, respectivamente, seguindo os padrões em voga nas universidades europeias, as disciplinas das áreas de Análise e de Geometria. No entanto, de acordo com os programas apresentados por Pires (2006), a presença de tópicos de Álgebra nesse curso era inicialmente reduzida - algo compreensível, uma vez que, como relatou Elza Furtado Gomide (1925-2013) em depoimento a Santos (2010, p. 68), "os italianos, sobretudo, se afastaram da Álgebra, [...] a Álgebra não se desenvolveu na Itália ao mesmo tempo que nos outros países, na Alemanha, por exemplo".

Esta área ganhou espaço no currículo pouco a pouco, a partir do momento em que a presença de integrantes do grupo Bourbaki se tornou constante na instituição, uma vez que, como já mencionado, estes foram fortemente influenciados pela abordagem que matemáticos alemães davam à Álgebra, evidenciada, por exemplo, em obras como a já citada Moderne algebra, de van der Waerden.

Gomide salienta, ainda no depoimento concedido a Santos (2010, p. 82), que esses professores exerceram influência marcante na mudança dos currículos. Introduziram, por exemplo, o ensino da Álgebra Linear, conteúdo que ela afirma não ter estudado e do qual nem sequer ouvira falar durante sua Graduação (1942-1945). 
A respeito da inserção, em razão dessa influência bourbakista, de novos conteúdos no curso de Matemática, Pires (2006, p. 341) ressalta que, inicialmente, ela se deu por meio de adaptações dos conteúdos originalmente trabalhados nas cadeiras já existentes. A autora afirma que, nesse período, “os limites destas cadeiras são ultrapassados e a inserção de tópicos apresentados por Bourbaki invadem-nas apesar do título que comportam”.

No que diz respeito à Álgebra Linear, foi introduzida não como disciplina, mas como conteúdo de ensino no âmbito da cadeira então chamada Complementos de Geometria e Geometria Superior. Para que tal inserção possa ser bem compreendida, apresentamos a seguir algumas informações referentes à criação dessa cadeira e aos conteúdos que originalmente eram nela trabalhados.

Como relata Pires (2006), Albanese era o responsável pela cadeira de Geometria. Com seu retorno à Itália, em abril de 1942, esta foi dividida em duas outras: Geometria Analítica Projetiva e Descritiva e Complementos de Geometria e Geometria Superior. Segundo informações presentes no Guia da Faculdade de Filosofia, Ciências e Letras (USP, 1943) 3 , os Complementos de Geometria eram ministrados conjuntamente, na $2^{\mathrm{a}}$ série, para os alunos dos cursos de Matemática e de Física. Já a Geometria Superior destinava-se apenas aos estudantes da $3^{\mathrm{a}}$ série do curso de Matemática. O programa para Complementos de Geometria, presente no Guia de 1943 era:

- Projetividade nas formas de III espécies. Classificação.

- Teoria das Quádricas. Sistemas de Quádricas. Quádricas Homofocais.

- Sistema Nulo e Geometria da Reta. Complexos e congruências de retas.

- O absoluto. Aplicações.

- Os entes racionais e o princípio da correspondência de Cremona Chasles.

- Projeção estereográfica e famílias de curvas sobre uma quádrica.

- Elementos de Topologia das Superfícies. Ordem de conexão. Superfícies biláteras e uniláteras. Fórmula de Euler generalizada. Gênero de uma superfície e moldes típicos. Aplicações: poliedros platônicos e poliedros eulerianos.

- Superfícies em geral. Ponto simples e pontos múltiplos. Planos e cones tangentes.

- Pontos elípticos, hiperbólicos e parabólicos.

- Comportamento do plano tangente nos vários casos.

- Superfícies regradas reversas e desenvolvíveis. Teorema de Chasles e P. Serret.

De acordo com Pires (2006), em 1942, Catunda era responsável por Complementos de Geometria e Cândido Lima da Silva Dias (1913-1998) o era por Geometria Superior. A partir de 1944, Dias assumiu interinamente a cadeira Complementos de Geometria e Geometria Superior, tendo como assistente Luiz Henrique Jacy Monteiro (1921-1975). Também segundo

\footnotetext{
${ }^{3}$ Foram consultados os Guias da FFCL de 1943, 1948, 1951, 1953, 1954 e 1965, disponíveis na biblioteca da Faculdade de Educação da Universidade de São Paulo.
} 
Pires (2006), em novembro de 1951, Dias prestou concurso para a cátedra Complementos de Geometria, tendo sido aprovado com o trabalho Espaços vetoriais e sua aplicação nos espaços funcionais analiticos. Pires (2006, p. 353) destaca que esse trabalho configura-se como "uma das primeiras manifestações de aceitação da perspectiva bourbakista da Matemática" e ressalta que Dias, por meio dessa obra, estabeleceu "uma ponte entre os funcionais analíticos apresentados pela escola tradicional italiana e os espaços vetoriais da escola bourbakista de Matemática francesa".

Embora os dados levantados para o presente artigo não nos permitam apontar com precisão quando os conteúdos de Álgebra Linear foram inseridos em Complementos de Geometria, eles nos possibilitam conjecturar que tal inserção ocorreu no início da década de 1950, já que, como evidenciam Duarte (2007) e Oliveira (2007), em 1952, o então graduando em Matemática pela FFCL da USP, Ubiratan D'Ambrosio (1932-), em Complementos de Geometria, ministrada pelo catedrático Dias e pelo assistente Jacy Monteiro, estudou Espaços Vetoriais, e não mais conteúdos que constavam no programa de 1943 para tal disciplina.

As fichas produzidas por D'Ambrosio com notas de aula desse curso foram analisadas por Duarte (2007), que as sintetizou em um quadro com os conteúdos nelas mencionados. Uma adaptação de tal quadro é apresentada na Figura 1.

\begin{tabular}{|c|}
\hline NOTAS DE AULA DE D'AMBROSIO (1952) \\
\hline $2^{\circ}$ ano \\
\hline $\begin{array}{l}\text { COMPLEMENTOS DE GEOMETRIA } \\
\text { (ESPAÇOS VETORIAIS) } \\
\text { Prof. Cândido Lima da Silva Dias }\end{array}$ \\
\hline $\begin{array}{l}\text { Vetor; espaço vetorial; dependência linear; } \\
\text { combinação linear; base; dimensão; } \\
\text { isomorfismo; subsepaço vetorial; itersecçăo } \\
\text { de subespaço; geração; soma de } \\
\text { subespaço; soma direta de subespaço; } \\
\text { dimensão da soma direta; forma linear; } \\
\text { espaço dual; forma bilinear fundamental; } \\
\text { dual de dual; isomorfismo canônico; } \\
\text { espaços reflexivos; anuladores; soma } \\
\text { direta. }\end{array}$ \\
\hline $\begin{array}{l}\text { Transformações lineares de um espaço em } \\
\text { outro; transformação inversa; matriz } \\
\text { associada a uma transformação linear; } \\
\text { redução completa de uma transformação; } \\
\text { transformação idempotente; involução; } \\
\text { sistemas lineares; característica; forma } \\
\text { canônica de uma matriz ou de Jordan. }\end{array}$ \\
\hline $\begin{array}{l}\text { Espaço vetorial normado. Espaço vetorial } \\
\text { completo. Transformaçōes lineares } \\
\text { definidas num espaço normado; } \\
\text { continuidade de um transformação; } \\
\text { transformação linear limitada; rotação. }\end{array}$ \\
\hline
\end{tabular}

Figura 1 - Conteúdos presentes nas fichas referentes produzidas por D'Ambrosio referentes às aulas de Complementos de Geometria assistidas em 1952

Fonte: Adaptado de Duarte (2007, p. 410). 
Como salienta Duarte (2007, p. 323), a bibliografia indicada nessa disciplina ministrada por Dias, em 1952, incluía as seguintes obras: Finite dimensional vector spaces, de Paul Halmos (1916-2006), de 1948; A survey of modern algebra, de Garrett Birkhoff e Saunders MacLane, de 1950; e Algèbre et analyse linéaires, de André Lichnerowicz (1915-1998), de 1947.

Convém destacar que os prefácios de todos esses manuais citados na bibliografia do curso salientam a importância da Álgebra Linear para aplicações tanto na própria Matemática, quanto em outras áreas do conhecimento. O mesmo se aplica à obra de Birkhoff e MacLane, já mencionada. Isso também pode ser observado ao analisarmos os prefácios dos textos de Halmos e de Lichnerowicz. O primeiro destaca que "o propósito é tratar transformações lineares em espaços vetoriais de dimensão finita pelos métodos de teorias mais gerais. A ideia é enfatizar as noções geométricas simples, comuns a muitas partes da Matemática e suas aplicações" (HALMOS, 1978, p. 9).

Na obra Algèbre et analyse linéaires, há um esforço, segundo o autor, em familiarizar o leitor com a álgebra dos operadores lineares e das matrizes, que, juntamente com a álgebra tensorial é fundamental para a compreensão de diferentes teorias da Física. No prefácio, redigido pelo matemático francês George Darmois (1888-1960), afirma-se que tal livro é uma iniciação às principais técnicas matemáticas da Física Moderna e evidencia-se o quanto a Matemática, em rápida evolução na época, havia se tornado indispensável para os físicos. Como destaca o físico teórico britânico Paul Dirac (1902-1984), em afirmação citada por Darmois em Lichnerowicz (1956), qualquer trabalho em Física Moderna não pode se limitar a uma mera descrição de fatos experimentais; exige um tratamento matemático.

Os dados que obtivemos nos permitem afirmar que, até 1964, a Álgebra Linear esteve presente no programa do curso de Matemática da FFCL da USP como conteúdo de ensino em Complementos de Geometria.

Somente em 1965 é que, pela primeira vez, aparece no currículo desse curso uma disciplina intitulada Álgebra Linear. Esta era ministrada na $2^{\mathrm{a}}$ série, ainda no âmbito da cadeira Complementos de Geometria e Geometria Superior, que nessa época estava sob a responsabilidade do professor catedrático Dias, do assistente Jacy Monteiro e do instrutor Roberto Celso Fabrício Costa (1941-).

Ana Maria Velloso Nobre (1945-), atualmente professora do Departamento de Matemática da Pontifícia Universidade Católica de São Paulo, relatou-nos, em conversa informal sobre pesquisa em desenvolvimento, haver cursado Álgebra Linear na graduação em Matemática, da FFCL da USP, em 1966 e nos possibilitou acesso ao principal material utilizado pelos estudantes em tal disciplina naquela época: o primeiro volume de um livro datilografado 
intitulado Álgebra Linear, de autoria de Jacy Monteiro, que, segundo Nobre, foi quem ministrou a disciplina na ocasião.

Analisando a introdução desse volume, datada de junho de 1965, notamos que a obra estava em sua terceira edição e que, "destinada aos cursos de Matemática das Faculdades de Filosofia, apresenta modificações em relação às edições anteriores, no sentido de uma maior simplificação da matéria exposta, visando mais os objetivos propostos no ensino da Álgebra Linear" (JACY MONTEIRO, 1965, s. num. p.).

Jacy Monteiro (1965) expõe que aquele primeiro volume visava proporcionar ao leitor um estudo geral dos espaços vetoriais, das aplicações lineares e das matrizes, sendo a exposição limitada aos espaços vetoriais de dimensão finita. Salienta alguns tópicos que, em sua visão, são essenciais em um primeiro estudo da Álgebra Linear, quais sejam: dependência linear, a noção de base de um espaço vetorial, o estudo das aplicações lineares, o cálculo matricial, a ideia de mudança de base, a introdução da forma normal de Hermite (em razão de sua aplicação ao problema da inversão de uma matriz quadrada) e a resolução de sistemas de equações lineares. Convém ressaltar que a bibliografia citada pelo autor no volume em questão fornece mais uma evidência da influência bourbakista no ensino da Álgebra Linear. As obras mencionadas incluem algumas às quais já nos referimos, como o capítulo 2 (intitulado Álgebra linear), do livro II (Álgebra), da primeira parte (As estruturas fundamentais da análise) dos Elementos de matemática, de Bourbaki; o tratado de Dieudonné, intitulado Algèbre linéaire et géométrie élémentaire; e o manual de Halmos, já utilizado por D’Ambrosio, em 1952.

A influência exercida pelos bourbakis quanto aos conteúdos matemáticos que pouco a pouco foram sendo inseridos aos currículos dos cursos não se restringiu à FFCL. Em diferentes ocasiões, professores dessa faculdade ministraram disciplinas também na Poli, levando tal influência também aos programas das disciplinas matemáticas presentes nas graduações em Engenharia ofertadas por essa escola.

Em relação especificamente à Álgebra Linear, destacamos o papel exercido por Alexandre Augusto Martins Rodrigues (1930-), que, segundo Calábria (2010), graduou-se em Matemática pela FFCL da USP, em 1952, e doutorou-se, em 1957, pela Universidade de Chicago, tendo sido orientado inicialmente pelo bourbaki André Weil. Iniciou sua carreira docente em 1952, como auxiliar de ensino da FFCL e, em 1959, foi contratado para reger a cátedra Geometria Analítica e Projetiva da Poli.

Rodrigues, conforme depoimento concedido a Dias (2002, p. 156), já atuava em 1958 como docente nessa cátedra e ministrou, pela primeira vez na Poli, o primeiro curso de Geometria Analítica a partir de um enfoque da Álgebra Linear. É a partir daí, portanto, que 
podemos nos referir à presença da Álgebra Linear como conteúdo de ensino nos cursos de Engenharia oferecidos nessa escola.

Tivemos acesso ao livro Álgebra linear e geometria euclidiana, publicado por Rodrigues, em 1966, e que, segundo o autor, foi redigido a partir das notas de aula dos cursos que ministrou na Poli, em 1959 e 1960, na cadeira Geometria Analítica. Esta obra evidencia, mais uma vez, a influência dos integrantes do grupo Bourbaki na seleção dos conteúdos matemáticos a serem trabalhados na USP, naquela época, e na maneira de ensiná-los. A abordagem de Geometria proposta por Rodrigues é a mesma defendida por Dieudonné, já presente na obra Algèbre linéaire et géométrie élémentaire deste último, de 1964.

$\mathrm{Na}$ introdução de seu livro, Rodrigues informa que serão apresentadas as noções básicas da teoria dos espaços vetoriais e que estas serão utilizadas para construir um modelo de Geometria Euclidiana. Ressalta que "da teoria dos espaços vetoriais abordamos somente aqueles tópicos que julgamos indispensáveis num primeiro contato com a Álgebra Linear e que, ao mesmo tempo, constituem o essencial para desenvolver a parte geométrica" (RODRIGUES, 1966, s. num. p.). Salienta também que serão considerados apenas espaços vetoriais de dimensão finita, sendo os escalares sempre números reais. Complementa que "essas restrições foram feitas visando maior simplicidade do texto, mas julgamos, de qualquer modo, que o leitor que tenha dominado a teoria nesse caso particular poderá com muito pouco trabalho adicional, enfronhar-se de situações mais gerais" (RODRIGUES, 1966, s. num. p.).

Na FFCL, o ingresso da Álgebra Linear como conteúdo de ensino se deu em cursos que adotavam como referências livros que enfatizavam a importância do estudo daquela área em razão de suas aplicações, especialmente na Física. Já na Poli, lócus que tendemos a ver como promotor de uma utilização mais prática dos conteúdos estudados, essa inserção decorreu de uma motivação essencialmente matemática, que Rodrigues evidencia na introdução de sua obra:

A Geometria Euclidiana pode ser introduzida ou com base nos postulados que remontam a Euclides e que, do ponto de vista de uma teoria axiomática, foram apresentados, pela primeira vez, em forma satisfatória por Hilbert, ou através da Geometria Projetiva, ou como caso muito particular de teorias abordadas em Geometria Diferencial ou ainda através da Álgebra Linear, onde o conceito de vetor desempenha papel fundamental. Não hesitamos em afirmar que, para o ensino da Geometria nos primeiros anos universitários, o melhor método e talvez mesmo o único viável é este último. [...] O ensino da Geometria através da Álgebra Linear é de tal modo simples, põe tão claramente em evidência a essência dos fenômenos geométricos e contribui tanto para a formação matemática do estudante, que não faltam hoje matemáticos, entre os mais ilustres, que propugnam a sua adoção mesmo no curso secundário. Por outro lado, a aplicação da Álgebra Linear à Geometria facilita ao estudante a compreensão e o domínio dessa teoria. Outra vantagem é que permite tratar os espaços de dimensão $n$ sem mais trabalho que o exigido para o caso de dimensão 2 ou 3. Embora nos limitemos a considerar somente aspectos 
fundamentais, acreditamos deixar claro ao leitor que chegue ao fim destas notas, que os nossos axiomas de espaço euclidiano permitem efetivamente construir um modelo da Geometria Euclidiana (RODRIGUES, 1966).

De acordo com informações presentes em Rodrigues, Oliva e Harle (1963) e em Santos (1985), a Álgebra Linear instaura-se efetivamente como disciplina na Poli em 1961, quando aquela até então denominada Elementos de Geometria Analítica e Projetiva ligada à cadeira Geometria Analítica e Projetiva, sob responsabilidade de Rodrigues e que desde 1958 contemplava a Álgebra Linear como conteúdo de ensino, passou a chamar-se Elementos de Álgebra Linear e Geometria Analítica.

Tal como na FFCL no que diz respeito à Álgebra Linear, na Poli a influência bourbakista teve início como adaptações dos conteúdos originalmente contemplados em uma disciplina já existente, até que esta fosse mais tarde oficialmente reorientada tanto em termos de conteúdos programáticos, quanto de nomenclatura.

\section{Reflexões finais}

Nesta pesquisa, consideramos a Álgebra Linear como uma disciplina acadêmica universitária, na concepção de Resende (2007). Como ressalta Lima (2012, p. 42, grifo do autor) a partir das ideias de Resende (2007) e de Pessoa (2007),

[...] esse tipo de disciplina inclui diversos elementos ligados às questões dos processos de ensino e de aprendizagem, tais como as finalidades do curso no qual está inserida, o perfil do profissional que se deseja formar, etc. [...] e diferencia-se da noção de disciplina escolar por ter como objetivo formar um profissional, seja ele, professor, cientista, administrador técnico, etc., e não a formação de um indivíduo comum, que necessita de instrumentos intelectuais múltiplos para se estabelecer na sociedade e utilizar a capacidade de compreender o mundo em diversos aspectos físicos, econômicos, sociais e culturais.

Apesar dessa diferença, pudemos observar, no processo de inserção da Álgebra Linear no Ensino Superior brasileiro, um aspecto que, segundo Chervel (1990), é característico da história das disciplinas escolares: há um momento de transição, neste caso iniciado pela introdução de noções de Álgebra Linear como conteúdos de ensino em disciplinas de Geometria já existentes (Complementos de Geometria, na FFCL, e Elementos de Geometria Analítica e Projetiva, na Poli). Ao mesmo tempo que o modelo antigo permanece, embora com algumas modificações, o novo começa a se instaurar.

Também em concordância com o que salienta Chervel (1990), pudemos perceber a renovação do corpo docente como fator determinante na evolução das disciplinas. No curso de Matemática, foi quando Cândido Lima da Silva Dias assumiu a disciplina Complementos de 
Geometria que esta começou a ser reorientada, até configurar-se como uma nova disciplina especificamente voltada à Álgebra Linear. Também nas engenharias da Poli, foi quando Alexandre Augusto Martins Rodrigues assumiu a cátedra Geometria Analítica e Projetiva que a disciplina atrelada a esta passou a contar, entre seus conteúdos programáticos, com noções de Álgebra Linear.

Ressaltamos também que, no caso específico do processo de inserção da Álgebra Linear no Ensino Superior brasileiro, essa renovação a que se refere Chervel (1990) pode ser entendida não como mera troca dos docentes responsáveis por determinadas disciplinas, mas sim como atualização do quadro de professores em termos de conhecimentos, consequência direta das atividades desenvolvidas por integrantes do grupo Bourbaki, que atuaram na USP da década de 1940 à de 1960.

Encerramos ressaltando a importância de se compreender o processo de inclusão da Álgebra Linear no Ensino Superior brasileiro. Tal compreensão nos possibilita analisar qual era, na época da implantação da disciplina nos currículos da Matemática e da Engenharia da USP, sua razão de ser.

Em consonância com a Teoria Antropológica do Didático (TAD), entendemos a razão de ser (BOSCH; GASCÓN, 2010) como sendo o porquê de ensinar determinado conteúdo, bem como os objetivos a alcançar com esse ensino. No caso de nossa investigação, trata-se de compreender por que a Álgebra Linear, na época de sua introdução nos currículos dos cursos mencionados, era entendida como necessária. Na FFCL, tal necessidade era percebida em função das aplicações da Álgebra Linear, à época cada vez mais frequentes, a diferentes áreas do conhecimento, com destaque para a Física. Na Poli, por sua vez, a Álgebra Linear foi introduzida visando possibilitar o ensino de Geometria Euclidiana de uma maneira alternativa e, na concepção do docente que estava à frente da disciplina na época, mais simples, que possibilitasse evidenciar a essência dos fenômenos geométricos.

\section{Referências}

BIRKHOFF, G.; MACLANE, S. A Survey of Modern Algebra. New York: Macmillan, 1941.

BOSCH, M.; GASCÓN, J. Fundamentación antropológica de las organizaciones didácticas: de los "talleres de prácticas matemáticas" a los "recorridos de estudio e investigación. In: Diffuser les mathématiques (et les autres savoirs) comme outils de connaissance et d'action, 2010. p. 49-85. Disponível em: $<$ http://www.atd-tad.org/wp-content/uploads/2012/05/mariannaJosep-CITAD-II2010.pdf>. Acesso em: 01 dez. 2017.

CALÁBRIA, A. R. Primeiro Colóquio Brasileiro de Matemática: identificação de um registro e pequenas biografias de seus participantes. 2010. 175 f. Dissertação (Mestrado em Educação Matemática) - Universidade Estadual Paulista, Rio Claro, 2010. 
CHERVEL, A. História das disciplinas escolares: reflexões sobre um campo de pesquisa. Teoria \& Educação, Palmarinca, n. 2, p. 177-229, 1990.

DIAS, A. L. M. Engenheiros, Mulheres, Matemáticos: Interesses e Disputas na Profissionalização da Matemática na Bahia (1896-1968). 2002. 320 f. Tese (Doutorado em História Social) - Faculdade de Filosofia, Letras e Ciências Humanas, Universidade de São Paulo, São Paulo, 2002.

DIEUDONNÉ, J. Algébre linéaire et geométrie élementaire. In: Collection Enseignement des Sciences. 4. ed. Paris: Hermann, 1973.

DIEUDONNÉ, J. A formação da Matemática Contemporânea. Trad. J. H. von Hafe Perez. Lisboa: Publicações Dom Quixote, 1990.

DORIER, J. L. Analyse historique de l'emergence des concepts elementaires d'algebre linéaire. In: Les cahiers de didactique des Mathematiques, Institut de Recherche pour L'Enseignement des Mathematiques, Universite, n.7, jun. 1990.

DORIER, J. L. On the Teaching of Linear Algebra. In: Mathematics Education Library. New York, Boston, Dordrecht, London, Moscow: Kluwer Academic Publishers, 2002. v. 23.

DUARTE, A. R. S. Matemática e Educação Matemática: a dinâmica de suas relações ao tempo do movimento da Matemática Moderna no Brasil. 2007. 438 f. Tese (Doutorado em Educação Matemática) - Pontifícia Universidade Católica de São Paulo, São Paulo, 2007.

GOUVÊA, F. Q. Resenha da obra Survey of Modern Algebra, 2010. Disponível em: $<$ https://www.maa.org/press/maa-reviews/a-survey-of-modern-algebra-0 $\geq$. Acesso em: 14 mar. 2018.

HALMOS, P. R. Espaços vetoriais de dimensão finita. Trad. de Guilherme de la Penha. Rio de Janeiro: Campus, 1978.

JACY MONTEIRO, L. H. Álgebra Linear, $1^{\text {o }}$ volume, 3. ed. São Paulo: Livraria Nobel S.A., 1965.

LICHNEROWICZ, A. Algèbre et analyse linéaires, 2a ed. Paris: Éditions Jacques Gabay, 1956.

LIMA, G. L. A Disciplina de Cálculo I do Curso de Matemática da Universidade de São Paulo: um estudo de seu desenvolvimento, de 1934 a 1994. 2012. 445 f. Tese (Doutorado em Educação Matemática) - Pontifícia Universidade Católica de São Paulo, São Paulo, 2012.

OLIVEIRA, M. C. A. A Formação Matemática de um Matemático e Educador Matemático. In: VALENTE, W. R. (Org.). Ubiratan D'Ambrosio: conversas; memórias; vida acadêmica; orientandos; educação matemática; etnomatemática; história da matemática; inventário sumário do arquivo pessoal. São Paulo: Annablume; Brasília: CNPq, 2007. cap. 2.

PESSOA, R. B. Um olhar sobre a trajetória da Geografia Escolar no Brasil e a visão dos alunos do Ensino Médio sobre a Geografia atual. 2007. 130f. Dissertação (Mestrado em Geografia) Universidade Federal da Paraíba, João Pessoa, 2007.

PIRES, R. C. A Presença de Nicolas Bourbaki na Universidade de São Paulo. 2006. 577 f. Tese (Doutorado em Educação Matemática) - Pontifícia Universidade Católica de São Paulo, São Paulo, 2006.

Ministére de L'Education Nationale de L'Enseignement Supérieur et de La Recherche. PROGRAMMES DU LYCEE. Disponível em: $<$ http://eduscol.education.fr/pid26017/programmeslycee.html $\geq$. Acesso em: 05 abr. 2017. 
RESENDE, M. R. Re-significando a disciplina Teoria dos Números na formação do professor de Matemática na Licenciatura. 2007. 281 f. Tese (Doutorado em Educação Matemática) - Programa de Estudos Pós-Graduados em Educação Matemática, Pontifícia Universidade Católica de São Paulo, São Paulo, 2007.

RODRIGUES, A. A. M. Álgebra Linear e Geometria Euclidiana. São Paulo: LPM Editora, 1966.

RODRIGUES, A. R; OLIVA, W. M.; HARLE, C. E. Geometria Analítica e Projetiva, Elementos de Álgebra Linear e Geometria Analítica: notas de aula da disciplina n. 21, durante os anos de 19601963. São Paulo: EPUSP, 1963.

SANTOS, A. A. Elza Furtado Gomide e a participação feminina no desenvolvimento da Matemática brasileira no século XX. 2010. 132 f. Dissertação (Mestrado em História da Ciência) Pontifícia Universidade Católica de São Paulo, São Paulo, 2010.

SANTOS, M. C. L. Escola Politécnica de São Paulo: 1894 - 1984. São Paulo: Reitoria da Universidade de São Paulo: Escola Politécnica: Fundação para o Desenvolvimento Tecnológico da Engenharia, 1985.

USP - UNIVERSIDADE DE SÃO PAULO. Faculdade de Filosofia, Ciências e Letras. Guia da Faculdade de Filosofia, Ciências e Letras para 1943. São Paulo: USP, 1943.

ZICCARDI, L. R. N. O Curso de Matemática da PUC/SP: uma história de sua construção/desenvolvimento/legitimação. 2009. 408 f. Tese (Doutorado em Educação Matemática) Pontifícia Universidade Católica de São Paulo, São Paulo, 2009.

Submetido em 07 de Dezembro de 2017. Aprovado em 12 de Maio de 2018. 